\title{
Gonadectomia pré-puberal em cães e gatos
}

\author{
Prepubertal gonadectomy in dogs and cats
}

\begin{abstract}
Fabiana Azevedo Voorwald ${ }^{\text {I* }}$ Caio de Faria Tiosso ${ }^{\mathrm{I}}$ Gilson Hélio Toniollo ${ }^{\mathrm{II}}$
\end{abstract}
\section{- REVISÃO BIBLIOGRÁFICA -}

\section{RESUMO}

A população de cães e gatos errantes é um problema grave de saúde pública e bem estar animal e a gonadectomia pré-puberal a partir de seis semanas de idade é a base para controle populacional efetivo. Os efeitos benéficos e maléficos são discutidos nesta revisão, auxiliando o médico veterinário a obter conhecimento e fundamentação cientifica para análise crítica do tema, propagação dessa prática e conscientização de proprietários.

Palavras-chave: gonadectomia, pré-pubere, superpopulação, esterilização cirúrgica.

\section{ABSTRACT}

Stray dogs and cats is a serious problem to public health and animal welfare. Prepubertal gonadectomy from six weeks of age is a solution to effective pet overpopulation control. The benefits and adverse effects of this procedure are discussed in this paper, allowing the veterinarians to obtain knowledge and scientific basis to critical analysis to the topic, practice spread and owner awareness.

Key words: gonadectomy, prepubertal, pet overpopulation, surgical sterilization.

\section{INTRODUÇÃO}

Tradicionalmente, no Brasil, a esterilização cirúrgica de cães e gatos é realizada a partir de seis meses de idade, quando se completa a primeira fase da maturidade física. Entretanto, pode ser realizada a partir da sexta semana de idade, como comumente praticado desde 1980, na Europa e Estados Unidos (SALMERI et al., 1991). O procedimento gera controvérsias entre os médicos veterinários, devido ao pouco conhecimento sobre dados científicos que justifiquem essa prática, sobre efeitos benéficos ou maléficos, sobre o adequado protocolo anestésico para o paciente pediátrico e sobre a técnica cirúrgica.

Objetiva-se discutir os benefícios e riscos da gonadectomia precoce, auxiliando o médico veterinário a obter conhecimento e fundamentação científica suficientes para análise crítica do tema, indicação dessa prática e conscientização de proprietários.

Efeitos benéficos e riscos da gonadectomia pré-puberal A população de cães e gatos errantes é um problema grave de saúde pública e bem estar animal. Em 1992, a American Humane Association proibiu a doação de animais inteiros nos Estados Unidos e, desde então, importantes organizações, responsáveis pelo bem-estar animal, endossam a gonadectomia a partir da sexta semana de idade (KUSTRITZ, 2002). $\mathrm{O}$ procedimento evita gestações indesejadas, reduz superpopulação de cães e gatos errantes, problemas causados por interação homem e animal, como transmissão de zoonoses, acidentes automobilísticos e ataques aos humanos, e possibilita reduzir

\footnotetext{
IPrograma de Pós-graduação em Cirurgia Veterinária, Faculdade de Ciências Agrárias e Veterinárias, (FCAV), Universidade Estadual Paulista (UNESP), Via de Acesso Prof. Paulo Donato Castelanne, s/n, 14884-900, Jaboticabal, SP, Brasil. E-mail: voorwald@gmail.com.

*Autor para correspondência.

IIDepartamento de Medicina Veterinária Preventiva e Reprodução Animal, FCAV, UNESP, Jaboticabal, SP, Brasil.
} 
contaminação e propagação de afecções transmitidas pela cópula, afecções congênitas ou hereditárias, auxiliar na estabilização de pacientes acometidos por doenças endócrinas e prevenir ou tratar afecções no trato reprodutor. Entretanto, os efeitos da castração precoce, a curto ou longo prazo, variam com a idade na qual o procedimento é realizado, sexo, espécie, raça e fatores ambientais.

\section{Complicações anestésicas e cirúrgicas}

Pacientes pediátricos podem ser submetidos a procedimento anestésico e cirúrgico, antes dos seis meses de idade, desde que suas particularidades fisiológicas sejam compreendidas e respeitadas (HOWE, 2006). Filhotes são suscetíveis a complicações como hipotermia, hipoglicemia, excitabilidade, sobredose de fármacos, parada cardiorrespiratória, regurgitação e aspiração (HOWE, 1997; KUSTRITZ, 2002). Entretanto, HOWE (1997) não verificou diferenças na incidência de complicações anestésicas em filhotes castrados com idade inferior a 12 semanas, entre 12 e 24 semanas, ou superior a 24 semanas.

As técnicas de gonadectomia descritas são semelhantes para pacientes pediátricos e adultos, mas a manipulação dos órgãos deve ser cuidadosa, devido à fragilidade tecidual, evitando-se quaisquer perdas sanguíneas e consequente hipotensão por hipovolemia (HOWE, 1997; KUSTRITZ, 2002). A castração nesses animais é mais simples e rápida, em comparação ao procedimento realizado póspuberdade, devido ao menor peso corpóreo, tamanho, quantidade de gordura e calibre dos vasos sanguíneos, possibilitando melhor visualização das gônadas, menor manipulação dos órgãos, menor sangramento, menor tempo operatório e incisão cirúrgica.

\section{Efeitos no trato reprodutor}

A ovariohisterectomia e ovariectomia são procedimentos profiláticos e terapêuticos para afecções mediadas por hormônios gonadais, como neoplasias em glândula mamária, útero, ovário, vagina e vulva, cistos ovarianos, pseudogestação, hiperplasia e prolapso vaginal/uterino, hiperplasia endometrial cística e muco/hemo/piometra. Em machos, a orquiectomia bilateral exerce efeito profilático e terapêutico para afecções andrógeno-dependentes, como hiperplasia prostática benigna, prostatite crônica, cistos e abscessos prostáticos/paraprostáticos, adenomas e hérnias perineais, desordens epididimárias e testiculares como neoplasia, torção do funículo espermático, orquite e epididimite.
Efeitos nas características sexuais secundárias

Os hormônios gonadais são fundamentais no desenvolvimento normal da genitália externa. Fêmeas caninas castradas até sete meses podem apresentar vulva menos desenvolvida (infantil), predispondo à dermatite perivulvar e vaginite (SALMERI et al., 1991; ROOT et al., 1997). Gatas castradas na pré-puberdade apresentam vulva infantil, mas não desenvolvem o problema clínico relacionado à inflamação do órgão (KUSTRITZ, 2007). A expectativa do primeiro estro para desenvolvimento da vulva não é comprovadamente eficaz, pois fêmeas castradas tardiamente podem apresentar atrofia vulvar a longo prazo (KUSTRITZ, 2002; REICHLER, 2009), e o controle de peso pode reduzir a predisposição à inflamação da vulva e vagina, consequente à vulva infantil.

Cães submetidos à orquiectomia precoce podem apresentar imaturidade no desenvolvimento do prepúcio e pênis e, em gatos, pode haver subdesenvolvimento ou ausência de espículas penianas (STUBBS et al., 1996), entretanto, essas alterações não interferem na qualidade de vida do animal.

A separação da prega balanoprepucial, dependente dos andrógenos testiculares, completa-se após a puberdade em felinos. ROOT et al. (1996b) verificaram incapacidade de exteriorização do pênis em $100 \%$ dos gatos castrados na pré-puberdade, $40 \%$ dos castrados aos sete meses e em nenhum gato inteiro. Entretanto, STUBBS et al. (1996) afirmaram que todos os gatos orquiectomizados, independente da idade, são capazes de expor o pênis. A falha na separação da prega balanoprepucial não é um problema clínico e não predispõe à doença do trato urinário inferior de felinos ou obstrução urinária (SPAIN et al., 2004b; HOWE, 2006), apenas dificulta a auto-higienização e a cateterização uretral nesses animais.

\section{Efeitos no metabolismo}

Ganho de peso

Felinos castrados, independente da idade, possuem menor coeficiente térmico, maior massa corpórea, escore corporal e quantidade de gordura falciforme, em comparação aos inteiros, e apresentam risco 3,4 vezes maior de se tornarem obesos, necessitando menor energia de manutenção; $33 \%$ menos calorias para fêmeas e $28 \%$ para machos (ROOT et al., 1996a; HOWE et al., 2000, KANCHUK et al., 2002; NGUYEN et al., 2004; SPAIN et al.; 2004b). Os estudos são controversos a respeito da obesidade em cães castrados. Fêmeas castradas apresentam maior ingestão de alimento, apetite indiscriminado e risco duas vezes maior de se tornarem obesas (O'FARRELL \& PEACHEY, 1990).

Ciência Rural, v.43, n.6, jun, 2013. 
HEIDENBERGER \& UNSHELM (1990) verificaram maior ingestão de alimentos em $42 \%$ dos machos e $32 \%$ das fêmeas castradas, resultando em decréscimo do nível de atividade e maior tempo de repouso. SPAIN et al. (2004a) encontraram maior prevalência de obesidade em fêmeas e machos castrados na idade tradicional, em comparação aos castrados antes dos cinco meses.

A obesidade é um problema multifatorial, influenciado por dieta, atividade física, raça, idade e condição sexual. O problema não ocorre exclusivamente em consequência à gonadectomia e os autores desta revisão evidenciaram em sua rotina influência negativa maior da castração pós-puberal, em comparação à pré-puberal, na prevalência de animais obesos. É comprovado que animais gonadectomizados, independente da idade, necessitam ingerir menor quantidade de alimentos após castração, portanto, tal efeito maléfico pode ser evitado com manejo adequado.

\section{Diabetes mellitus (DM)}

HOENIG \& FERGUSON (2002) verificaram resistência transitória à insulina em gatas castradas e McCANN et al. (2007) e PRAHL et al. (2007) demonstraram que felinos castrados apresentam risco 8,7 vezes maior de desenvolver DM, associado à elevação nas concentrações plasmáticas de insulina secundária ao maior consumo de alimentos. Acredita-se que o aumento na prevalência de DM em felinos castrados relaciona-se ao aumento do peso corpóreo, e não diretamente ao efeito da privação dos hormônios gonadais no metabolismo da insulina, portanto, pode ser evitado com redução na oferta de alimentos. SCOTT-MONCRIEFF (2010) sugeriram redução da ligação à insulina e transporte de glicose aos tecidos em gatas durante diestro e gestação, sob influência da progesterona.

Em cadelas inteiras, altas concentrações de progesterona $\left(\mathrm{P}_{4}\right)$, durante o diestro e gestação, exercem efeito antagônico crônico à insulina, reduzem sua ligação e o transporte de glicose aos tecidos alvos (PÖPPL et al., 2007), estimulam a produção de hormônio de crescimento pelo epitélio ductal hiperplásico nas glândulas mamárias, desestabilizam o controle glicêmico e resultam em intolerância à glicose e resistência insulínica (SCARAMAL et al., 1997; REICHLER, 2009). Portanto, a gonadectomia, independente da idade, pode ser considerada importante medida profilática para o desenvolvimento, evolução e progressão da DM, em cadelas e gatas.

\section{Hiperadrenocorticismo e hipotireoidismo}

Ferrets castrados apresentam maior prevalência de afecções nas glândulas adrenais, em comparação aos inteiros, resultante de redução nos hormônios gonadais, aumento nos hormônios gonadotróficos e consequente hiperplasia glandular (SCHOEMAKER et al., 2000; JOHNSONDELANEY, 2006). De maneira contraditória, em cadelas, alta concentração de $\mathrm{P}_{4}$ durante o diestro, eleva a secreção de cortisol pela adrenal em resposta ao $\mathrm{ACTH}$, predispondo ou agravando o hiperadrenocorticismo (REIMERS et al., 1990). Não existem estudos sobre a privação dos hormônios gonadais na adrenal de cães e gatos machos, e o efeito benéfico em fêmeas foi comprovado, portanto, o aumento no risco de hiperadrenocorticismo após castração em ferrets não deve ser extrapolado para cães e gatos.

Em estudo retrospectivo, PANCIERA (1994) afirma que cães castrados apresentam risco três vezes maior de desenvolver hipotireoidismo, resultante de tireoidite linfocítica autoimune, justificando influência negativa do procedimento sobre o sistema imune do animal. Os hormônios gonadais não exercem influência direta nas funções da tireóide (REIMERS et al., 1990) e DIXON et al. (1999) não encontraram correlação entre gonadectomia e hipotireoidismo em cães.

Efeitos no sistema imune

KELLY (1980) sugere que os procedimentos anestésicos e cirúrgicos não influenciam na resposta imune humoral, mas sim, na resposta imune celular à vacinação em cães e gatos, reduzindo a capacidade de resposta a agentes infecciosos, no pós-operatório. HOWE et al. (2001) e SPAIN et al. (2004a) verificaram maior prevalência de parvovirose em cães castrados antes de sete meses, em comparação aos castrados após sete meses, mas CRENSHAW \& CARTER (1995) não encontraram diferença na prevalência de parvovirose, coronavirose e cinomose, comparando-se castrados pré com póspuberdade. Em gatos, a gonadectomia pré-puberal não está relacionada com aumento na prevalência de doenças infecciosas (STUBBS et al., 1996; HOWE et al., 2000; SPAIN et al., 2004b).

Os estudos que demonstraram maior risco de doenças infecciosas após gonadectomia prépuberal (HOWE et al., 2001; SPAIN et al., 2004a) foram realizados com animais de abrigos; expostos a ambiente suscetível à propagação de agentes. Além disso, a comparação limitou-se à idade do animal no momento da gonadectomia e não foram consideradas prevalência e suscetibilidade em filhotes inteiros. 
Efeitos no sistema musculoesquelético

Retardo no fechamento das epífises ósseas

Os hormônios gonadais associados ao hormônio de crescimento são responsáveis por maturação e fechamento da cartilagem epifisária dos ossos longos (KUSTRITZ, 2002; SYED \& KHOSLA, 2005). O fechamento das epífises ósseas distais é retardado em cães e gatos submetidos à gonadectomia até sete meses de idade (SALMERI et al., 1991; STUBBS et al., 1996; SONTAS \& EKICI, 2007) e o período de proliferação e hipertrofia dos condrócitos é mais longo, resultando em maior crescimento ósseo longitudinal (13\% em fêmeas e $9 \%$ em machos) (ROOT et al., 1997). SALMERI et al. (1991) afirmaram que existe correlação inversamente proporcional entre a idade da gonadectomia e a altura de cães e gatos (castrados com sete semanas $>$ sete meses $>$ castrados após fechamento epifisário ou animais inteiros).

Densidade óssea

A deficiência de hormônios gonadais em mulheres após menopausa ou submetidas à ovariectomia resulta em decréscimo da densidade óssea (RIGGS et al., 2002). A ausência de estradiol $\left(E_{2}\right)$ aumenta a taxa de remodelação, prolonga a fase de reabsorção e reduz a fase de formação óssea (RIGGS et al., 2002) e a $\mathrm{P}_{4}$ previne a perda óssea, mediada por receptores de $\mathrm{P}_{4}$ nos osteoblastos (DINNY GRAHAM \& CLARKE, 1997). SHEN et al. (1992) e EKICI et al. (2005; 2007) encontraram conteúdo mineral ósseo e densidade mineral óssea semelhantes em filhotes castrados antes, após cinco meses ou inteiros.

\section{Fraturas epifisárias}

MAY (1998) e McNICHOLAS et al. (2002) sugeriram que o retardo no fechamento epifisário, em animais castrados precocemente, favorece ocorrência de fraturas epifisárias de Salter-Harris proximais; todavia, os animais acometidos apresentavam sobrepeso. McNICHOLAS et al. (2002) e FISCHER et al. (2004) propuseram quatro fatores de risco para este tipo de fratura: gênero, status reprodutivo, retardo no fechamento epifisário e obesidade. Porém, SALMERI et al. (1991), SPAIN et al. (2004a,b) e SONTAS \& EKICI (2007) afirmaram que não existe correlação entre idade da gonadectomia e fraturas epifisárias.

Displasia coxofemoral (DCF) e Ruptura de ligamento cruzado cranial (RLCC)

Sugere-se que o maior comprimento dos ossos longos de animais castrados precocemente altera o ângulo e a conformação das articulações, predispondo à DCF e ruptura de ligamento cruzado cranial (SPAIN et al., 2004a). VAN HAGEN et al. (2005) afirmaram que cães gonadectomizados apresentam risco 1,5 vezes maior de DCF, mas os autores não consideraram o peso do animal e a idade à gonadectomia, sugerindo possível correlação com obesidade. SPAIN et al. (2004a) verificaram aumento na prevalência de DCF em cães castrados antes dos 5,5 meses $(6,7 \%)$, em comparação àqueles cães castrados entre 5,5 meses e um ano de idade (4,7\%), mas os animais acometidos, castrados pré-puberdade, apresentavam forma menos severa de DCF, corroborando os achados de HOWE et al. (2001).

SLAUTERBECK et al. (2004) relataram que cães castrados apresentam risco duas vezes maior de RLCC, mas os autores não consideraram fatores como peso do animal ou idade à gonadectomia. Autores sugerem que o crescimento prolongado após gonadectomia pré-puberal não predispõem à DCF e RLCC e fatores hereditários, raça, peso e escore corporal devem ser considerados (KUSTRITZ, 2007).

De fato, verifica-se maior crescimento ósseo longitudinal em animais castrados prépuberdade, mas não existem estudos que comprovem as implicações do retardo no fechamento epifisário, com crescimento assincrônico de ossos longos e, subsequente, má conformação articular. Sugerese que o crescimento prolongado ocorra de forma proporcional em todos os ossos, e que a ausência de hormônios gonadais não interfere na densidade óssea normal desses animais. Portanto, a castração pré-puberal parece não aumentar a predisposição a afecções musculoesqueléticas, em cães e gatos.

\section{Osteossarcoma}

RU et al. (1998) afirmaram que cães castrados apresentam risco duas vezes maior de desenvolverem osteossarcoma, mas não considerou a idade dos animais à gonadectomia. COOLEY et al. (2002) verificaram que cães machos da raça Rottweiler, castrados antes de um ano de idade, apresentaram risco quatro vezes maior e fêmeas três vezes maior de desenvolverem osteossarcoma, em comparação aos inteiros, contudo, KUSTRITZ (2007) questionou o estudo e sugere componente hereditário para a alta predisposição de sarcoma ósseo nessa raça. Pouco se sabe sobre a influência dos hormônios gonadais na carcinogênese óssea, mas COMPSTON (2001) sugeriu que $E_{2}$ e testosterona desempenham função de agentes pró-diferenciação, que inibem a transformação maligna de osteoblastos. 
O osteossarcoma é uma neoplasia mesenquimal, que acomete principalmente o esqueleto apendicular de cães de raças grandes e gigantes, com rápido crescimento ósseo. Visto que diferentes autores confirmam maior crescimento ósseo longitudinal em animais castrados antes da puberdade, sugere-se que os animais pré-dispostos ao osteossarcoma sejam castrados logo após a puberdade, até que novos estudos esclareçam a influência dos hormônios gonadais na carcinogênese óssea.

\section{Desenvolvimento muscular}

Acredita-se que a gonadectomia resulte em redução de tônus e massa muscular por redução dos efeitos anabólicos dos esteróides gonadais, que estimulam a fixação de nitrogênio nos músculos, promovendo crescimento e desenvolvimento de massa muscular por maior síntese proteica (SHAHIDI, 2001). Camundongos machos castrados apresentaram retardo no desenvolvimento muscular, em comparação aos inteiros (IGWEBUIKE, 2002), e a suplementação de testosterona, em homens hipogonadais/eugonadais, aumentou massa magra, força e desenvolvimento muscular (BHASIN et al., 2001). Não foram encontrados estudos a respeito dessas alterações em cães e gatos, mas observa-se maior deposição de gordura e menor tônus e massa muscular em cães e gatos castrados, independente da idade.

Efeitos no trato urinário inferior

Incontinência urinária

Acredita-se que a etiologia da incontinência urinária após gonadectomia seja multifatorial, envolvendo decréscimo na pressão de fechamento do esfíncter uretral, alterações hormonais relacionadas ao GnRH, FSH, LH e $E_{2}$, aumento na deposição de colágeno na musculatura lisa da bexiga, redução na contratilidade do músculo detrusor e na resposta aos estímulos elétricos e ao carbacol (ARNOLD et al., 1989; NICKEL, 1998; REICHLER et al., 2005; 2006; COIT et al., 2008). O risco de desenvolvimento da incontinência urinária é de 5,1\% (THRUSFIELD et al., 1998; ANGIOLETTI et al., 2004) a 20\% em fêmeas caninas castradas (ARNOLD et al., 1989) e menos de $1 \%$ em fêmeas inteiras, sendo raramente relatada em machos castrados ou inteiros (COIT et al., 2008). O efeito da idade ao procedimento sobre a prevalência da afecção ainda é controverso, SPAIN et al. (2004a) demonstraram frequência de 12,9\% e $5 \%$ de incontinência em fêmeas submetidas à gonadectomia antes e após os três meses de idade, respectivamente. HOWE et al. (2001) afirmaram não haver diferença significativa na prevalência dessa disfunção urinária após gonadectomia antes ou após o primeiro estro, e STÖCKLIN-GAUTSCHI et al. (2001) demonstraram menor incidência em fêmeas castradas até sete meses $(9,7 \%)$, em comparação às castradas após a puberdade $(21 \%)$.

Sabe-se que existe influência da ausência dos hormônios gonadais na ocorrência de incontinência urinária em cadelas, principalmente em raças grandes, mas os estudos sobre a idade durante gonadectomia, na predisposição à incontinência, ainda são controversos. Portanto, os autores deste trabalho não recomendam adiar o procedimento, objetivando evitar essa disfunção urinária.

Doença do trato urinário inferior de felinos (DTUI)

A percepção comum de que felinos são predispostos à obstrução uretral consequente à gonadectomia não é comprovada por dados científicos. Autores afirmaram que gatos castrados até sete meses de idade não apresentaram maior prevalência de obstrução do trato urinário, alterações no diâmetro uretral pré-prostático e peniano ou no perfil da pressão uretral, em comparação aos inteiros (SALMERI et al., 1991; ROOT et al., 1996b; STUBBS et al., 1996; SPAIN et al., 2004b). HOWE et al. (2000) encontraram maior prevalência de infecção urinária e obstrução uretral em gatos castrados após sete meses, em comparação aos castrados precocemente, e LEKCHAROENSUK et al. (2001) verificaram maior prevalência em felinos castrados, independente da idade, em comparação aos inteiros. Acredita-se que a maior prevalência de DTUI verificada em felinos castrados está relacionada com alterações ambientais e comportamentais, como redução de atividade, maior tempo de repouso e maior consumo de alimentos, resultante da ausência dos hormônios gonadais. Tais alterações podem ser evitadas com manejo adequado dos pacientes.

\section{Neoplasias}

Hemangiossarcoma e Carcinoma de células de transição da bexiga (CCT)

Autores verificaram maior risco de desenvolvimento de hemangiossarcoma cardíaco e esplênico em animais castrados, em comparação aos inteiros (PRYMAK et al., 1988; WARE \& HOPPER, 1999). NORRIS et al. (1992) e KNAPP et al. (2000) afirmaram que a gonadectomia em cães aumenta em duas a quatro vezes o risco de desenvolvimento de CCT em fêmeas e machos. Entretanto, os autores não consideraram a idade do animal na gonadectomia, e tais resultados devem ser associados à maior expectativa de vida dos animais castrados e, consequente, maior prevalência de afecções relacionadas à senilidade. 
Neoplasia prostática

Autores afirmaram que cães castrados apresentaram risco 3,9 vezes maior de desenvolverem neoplasia prostática em comparação aos inteiros (SORENMO et al., 2003; BRYAN et al., 2007), visto que a interrupção do estímulo androgênico gonadal reduz a proporção dos componentes acinares glandulares andrógeno-dependentes, resultando em aumento da proporção dos componentes ductais e uroteliais andrógeno-independentes, responsáveis pela neoplasia (RHODES et al., 2000; LEAV et al., 2001). Contrariamente, outros autores sugerem que a exposição aos hormônios testiculares contribui para a vulnerabilidade da próstata ao desenvolvimento neoplásico, e que a gonadectomia pós-puberdade não previne nem aumenta a prevalência de neoplasia prostática (TESKE et al., 2002), mas o procedimento pode agravar a progressão, a agressividade (CORNELL et al., 2000; TESKE et al., 2002) e a evolução metastática da doença existente (BELL et al., 1991).

Os autores deste trabalho acreditam que a gonadectomia precoce auxilia na prevenção de prostatopatias e neoplasia prostática em cães, visto que tais afecções ocorrem principalmente em tecidos que respondem de forma descontrolada à influência androgênica.

Neoplasia mamária

A manutenção da atividade gonadal de fêmeas é o maior risco para desenvolvimento de neoplasias mamárias, que representam 50\% dos tumores em cadelas e $17 \%$ em gatas (MORRISSON, 1998). O efeito proliferativo no epitélio glandular mamário, exercido pelos esteroides ovarianos, cria condições para a proliferação neoplásica e carcinogênese (SORENMO et al., 2000; CHANG et al., 2009). O risco é reduzido para $0,5 \%$ em cadelas castradas antes do primeiro estro, $8 \%$ após o primeiro estro e $26 \%$ após duas ou mais fases de estro, até os dois anos de idade (SCHNEIDER et al., 1969). Estudos demonstraram que a castração realizada antes do quarto ciclo estral exerce efeito protetor significativo contra neoplasia mamária e que, entre quatro e seis ciclos estrais, o efeito protetor ocorre, mas não é estatisticamente significativo (MISDORP, 1988; SONNENSCHEIN et al., 1991). RUTTEMAN et al. (2001) demonstraram que a castração após 2,5 anos de idade não reduz a incidência de neoplasias malignas, mas sim das benignas. Em gatas, o risco de desenvolvimento de neoplasia mamária é reduzido para $9 \%$ quando a gonadectomia é realizada antes de seis meses de idade, $14 \%$ antes de um ano e $89 \%$ entre um e dois anos de idade (OVERLEY et al., 2005).
O benefício da gonadectomia precoce na prevenção da neoplasia mamária é bem comprovado por dados científicos. Os autores verificaram, na rotina clínica, efeito protetor dose-dependente da ablação dos ovários no desenvolvimento tumoral mamário e, por isso, recomendam que o procedimento seja realizado nos primeiros anos de vida da paciente. Entretanto, ressalta-se que a ovariectomia resulta em aumento na sobrevida da fêmea acometida por tumores benignos ou malignos, até mesmo se realizada concomitantemente à mastectomia.

\section{Comportamento}

Diversos estudos sobre os efeitos da gonadectomia no comportamento de cães e gatos estão disponíveis e apresentam resultados variados. $\mathrm{Na}$ maioria dos casos, cães submetidos à gonadectomia pré ou pós-puberal apresentaram redução no comportamento de marcação territorial, fuga, monta em cães ou pessoas, agressividade, perambulação, ansiedade de separação e micção indesejada por medo, além de cães castrados obesos apresentarem grau de atividade reduzido e maior tempo de repouso (SALMERI et al., 1991; STUBBS et al., 1996; MAARSCHALKERWEERD et al., 1997; NIELSEN et al., 1997; SPAIN et al., 2004a).

A maioria dos gatos submetidos à orquiectomia pré ou pós-puberal apresentaram redução na agressividade, perambulação, marcação territorial com spray de urina (ROOT et al., 1996a; STUBBS et al., 1996; HOWE et al., 2000; SPAIN et al., 2004a,b) e aumento na afetividade com humanos (STUBBS et al., 1996). Ressalta-se que comportamentos indesejados como marcação territorial, fuga e agressividade, reduzem de forma satisfatória após gonadectomia pré-puberal, e que maior tempo de repouso e redução do grau de atividade são observados apenas nos animais que se tornam obesos, passíveis de serem submetidos a um manejo adequado.

\section{CONCLUSÃo}

Diferentes estudos comprovaram que animais castrados apresentam maior expectativa de vida, em comparação aos inteiros, resultante dos efeitos profiláticos e terapêuticos da gonadectomia em relação às afecções do trato reprodutor e da redução dos comportamentos de risco. Deve-se considerar que a maioria dos efeitos maléficos e riscos da gonadectomia ocorre de forma semelhante, quando o procedimento é realizado em idade pré-puberal ou póspuberal, que alguns dos efeitos supracitados resultam

Ciência Rural, v.43, n.6, jun, 2013. 
da maior expectativa de vida dos animais castrados e consequente aumento na predisposição a afecções comuns na senilidade. Além disso, a baixa ocorrência de efeitos colaterais pode ser insignificante em comparação à elevada prevalência de afecções sobre as quais o procedimento exerce efeito profilático. Portanto, é recomendado que médicos veterinários procedam com segurança à gonadectomia prépuberal em cães e gatos a partir da sexta semana de idade, respeitando-se as características individuais e fisiológicas dos pacientes.

\section{AGRADECIMENTOS}

Os autores agradecem à valiosa colaboração do Prof. Dr. Jeffrey Frederico Lui e à Associação Protetora dos Animais de Jaboticabal, SP.

\section{REFERÊNCIAS}

ANGIOLETTI, A. et al. Urinary incontinence after spaying in the bitch: incidence and oestrogen-therapy. Vet Res Commun, v.28, p.153-155, 2004. Disponível em: <http://link.springer.com/arti cle/10.1023\%2FB\%3AVERC.0000045394.31433.9e?LI=true $>$. Acesso em: 13 dez. 2012. doi: 10.1023/B:VERC.0000045394.3 1433.9e.

ARNOLD, S. et al. Urinary incontinence in spayed bitches: prevalence and breed predisposition. Schweiz Arch Tierheilk, v.131, p.259-263, 1989.

BELL, F.W. et al. Clinical and pathologic features of prostatic adenocarcinoma in sexually intact and castrated dogs: 31 cases. J Am Vet Med Assoc, v.199, p.1623-1630, 1991.

BHASIN, S. et al. Proof of the effect of testosterone on skeletal muscle. J Endocrinol, v.170, p.27-38, 2001. Disponível em: $<$ http://joe.endocrinology-journals.org/content/170/1/27.full.pdf $>$. Acesso em: 13 dez. 2012. doi: 10.1677/joe.0.1700027.

BRYAN, J.N. et al. A population study of neutering status as a risk factor for canine prostate cancer. Prostate, v.67, p.11741181, 2007. Disponível em: <http://onlinelibrary.wiley.com/ doi/10.1002/pros.20590/pdf>. Acesso em: 13 dez. 2012. doi: $10.1002 /$ pros. 20590 .

CHANG, C.C. et al. Evaluation of hormone receptor expression for use in predicting survival of female dogs with malignant mammary gland tumors. J Am Vet Med Assoc, v.235, p.391396, 2009. Disponível em: <http://avmajournals.avma.org/doi/ pdf/10.2460/javma.235.4.391>. Acesso em: 13 dez. 2012. doi: 10.2460/javma.235.4.391.

COIT, V.A. et al. Neutering affects urinary bladder function by different mechanisms in male and female dogs. Eur J Pharm, v.584, p.153-158, 2008. Disponível em: $<$ http://www.sciencedirect. com/science/article/pii/S0014299908001465>. Acesso em: 13 dez. 2012. doi: 10.1016/j.ejphar.2008.02.037.

COMPSTON, J.E. Sex steroids and bone. Physiol Rev, v.81, n.1, p.420-447, 2001. Disponível em: <http://physrev.physiology. org/content/81/1/419.full.pdf $>$. Acesso em: 13 dez. 2012. doi: 10.1210/jc.2002-021420.
COOLEY, D.M. et al. Endogenous gonadal hormone exposure and bone sarcoma risk. Cancer Epidemiol Biomarkers Prev, v.11, p.1434-1440, 2002. Disponível em: <http://cebp.aacrjournals.org/ content/11/11/1434.full.pdf $>$. Acesso em: 13 dez. 2012.

CORNELL, K.K. etal. Clinical and pathological aspects of spontaneous canine prostate carcinoma: A retrospective analysis of 76 cases. Prostate, v.45, p.173-183, 2000. Disponível em: $<$ http://onlinelibrary. wiley.com/doi/10.1002/1097-0045(20001001)45:2\%3C173::AIDPROS12\%3E3.0.CO;2-R/pdf $>$. Acesso em: 13 dez. 2012. doi: 10.1002/1097-0045(20001001)45:2<173::AID-PROS12>3.3.CO;2-I.

CRENSHAW, W.E.; CARTER, C.N. Should dogs in animal shelters be neutered early? Vet Med, v.90, p.756-760, 1995.

DINNY GRAHAM, J. et al. Physiological action of progesterone in target tissues. Endocr Rev, v.18, n,4, p.502-519, 1997. Disponível em: <http://edrv.endojournals.org/content/18/4/502. full.pdf $>$. Acesso em: 13 dez. 2012. doi: 10.1210/er.18.4.502.

DIXON, R.M. et al. Epidemiological, clinical, haematological and biochemical characteristics of canine hypothyroidism. Vet Rec, v.145, p.481-487, 1999. Disponível em: <http://veterinaryrecord. bmj.com/content/145/17/481.full.pdf>. Acesso em: 15 dez. 2012. doi: $10.1136 /$ vr.145.17.481.

EKICI, H. et al. Effect of prepubertal ovariohysterectomy on bone mineral density and bone mineral content in puppies. Acta Vet Hung, v.53, n.4, p.469-478, 2005. Disponível em: $<$ http://www. akademiai.com/content/fq1656h3un077704/?genre $=$ article $\&$ id $=\mathrm{d}$ oi\%3a10.1556\%2fAVet.53.2005.4.7>. Acesso em: 13 dez. 2012. doi: 10.1556/AVet.53.2005.4.7.

EKICI, H. et al. The effect of prepubertal ovariohysterectomy on spine 1 mineral density and mineral content in puppies: a preliminary study. Res Vet Sci, v.82, p.105-109, 2007. Disponível em: <http://www.sciencedirect.com/science/article/pii/ S0034528806001238>. Acesso em: 13 dez. 2012. doi: 10.1016/j. rvsc.2006.07.001.

FISCHER, H.R. et al. Surgical reduction and stabilization for repair of femoral capital physeal fractures in cats: 13 cases. J Am Vet Med Assoc, v.224, n.9, p.1478-1482, 2004. Disponível em: $<$ http:// avmajournals.avma.org/doi/pdf/10.2460/javma.2004.224.1478>. Acesso em: 18 dez. 2012. doi: 10.2460/javma.2004.224.1478.

HEIDENBERGER, E.; UNSHELM, J. Changes in the behavior of dogs after castration. Tierärztl Prax, v.18, p.69-75, 1990.

HOENIG, M.; FERGUSON, D.C. Effects of neutering on hormonal concentrations and energy requirements in male and female cats. Am J Vet Res, v.63, p.634-639, 2002. Disponível em: $<$ https://www.avma.org/News/Journals/Collections/Documents/ ajvr_63_5_634.pdf>. Acesso em: 13 dez. 2012. doi: 10.2460/ ajvr.2002.63.634.

HOWE, L.M. Short-term results and complications of prepubertal gonadectomy in cats and dogs. J Am Vet Med Assoc, v.211, p.5762, 1997.

HOWE, L.M. et al. Long-term outcome of gonadectomy performed at an early age or traditional age in cats. J Am Vet Med Assoc, v.217, n.11, p.1661-1665, 2000. Disponível em: <https:/www.avma.org/News/Journals/Collections/Documents/ javma_217_11_1661.pdf $>$. Acesso em: 13 dez. 2012. doi: 10.2460/javma.2000.217.1661. 
HOWE, L.M. et al. Long-term outcome of gonadectomy performed at an early age or traditional age in dogs. J Am Vet Med Assoc, v.218, n.2, p.217-221, 2001. Disponível em: <https://www.avma. org/News/Journals/Collections/Documents/javma_218_2_217. pdf>. Acesso em: 13 dez. 2012. doi: 10.2460/javma.2001.218.217.

HOWE, L.M. Surgical methods of contraception and sterilization. Theriog, v.66, p.500-509, 2006. Disponível em: <http://www. sciencedirect.com/science/article/pii/S0093691X06002317> Acesso em: $13 \mathrm{dez} .2012$. doi: 10.1016/j.theriogenology.2006.04.005.

IGWEBUIKE, U.M. Retardation of muscle growth in castrated male mice: further evidence for hormonal influence on muscle development. Niger Vet J, v.23, n.1, p.47-53, 2002. Disponível em: <http://www.ajol.info/index.php/nvj/article/ download/3447/11515>. Acesso em: 13 dez. 2012. doi: 10.4314/ nvj.v23i1.3447.

JOHNSON-DELANEY, C.A. Ferret adrenal disease: 2006 perspective. Exotic DVM, v.8, p.31-34, 2006.

KANCHUK, M.L. et al. Neutering induces changes in food intake, body weight, plasma insulin and leptin concentrations in normal and lipoprotein lipase-deficient male cats. J Nutr, v.132, suppl.2, p.1730-1732, 2002. Disponível em: <http://jn.nutrition.org/ content/132/6/1730S.full.pdf $>$. Acesso em: 14 dez. 2012.

KELLY, G.E. The effect of surgery on dogs on the response to concomitant distemper vaccination. Aust Vet J, v.56, p.556557, 1980. Disponível em: <http://onlinelibrary.wiley.com/ doi/10.1111/j.1751-0813.1980.tb02591.x/pdf>. Acesso em: 14 dez. 2012. doi: 10.1111/j.1751-0813.1980.tb02591.x.

KUSTRITZ, M.V.R. Early spay-neuter: clinical considerations. Clin Tech Small Anim Pract, v.17, n.3, p.124-128, 2002. Disponível em: <http://www.tc.umn.edu/ rootk001/early_spay_ neuter_article.pdf $>$. Acesso em: 14 dez. 2012. doi: 10.1053/ svms.2002.34328.

KUSTRITZ, M.V.R. Determining the optimal age for gonadectomy of dogs and cats. J Am Vet Med Assoc, v.231, n.11, p.1665 1675, 2007. Disponível em: <http://avmajournals.avma.org/doi/ pdf/10.2460/javma.231.11.1665>. Acesso em: 14 dez. 2012. doi: 10.2460/javma.231.11.1665.

LEAV, I. et al. Role of canine basal cells in prostatic postnatal development, induction of hyperplasia, sex hormone-stimulated growth; and the ductal origin of carcinoma. Prostate, v.47, p.149-163, 2001. Disponível em: <http://onlinelibrary.wiley. com/doi/10.1002/pros.1058/pdf>. Acesso em: 14 dez. 2012. doi: $10.1002 /$ pros. 1058

LEKCHAROENSUK, C. et al. Epidemiologic study of risk factors for lower urinary tract diseases in cats. J Am Vet Med Assoc, v.218, p.1429-1435, 2001. Disponível em: <http://avmajournals. avma.org/doi/pdfplus/10.2460/javma.2001.218.1429>. Acesso em: 14 dez. 2012. doi: 10.2460/javma.2001.218.1429.

MAARSCHALKERWEERD, R.J. et al. Influence of orchiectomy on canine behaviour. Vet Rec, v.140, n.24, p.617619, 1997. Disponível em: <http://veterinaryrecord.bmj.com/ content/140/24/617.full.pdf $>$. Acesso em: 14 dez. 2012. doi: 10.1136/vr.140.24.617

MAY, C. Orthopaedic effects of prepubertal neutering in dogs. Vet Rec, v.142, p.71-72, 1998.
McCANN, T.M. et al. Feline diabetes mellitus in the UK: the prevalence within an insured cat population and a questionnairebased putative risk factor analysis. J Feline Med Surg, v.9 p.289-299, 2007. Disponível em: <http://jfm.sagepub.com/ content/9/4/289.full.pdf $>$. Acesso em: 14 dez. 2012. doi: 10.1016/j.jfms.2007.02.001.

McNICHOLAS, W.T. et al. Spontaneous femoral capital physeal fractures in adult cats: 26 cases (1996-2001). J Am Vet Med Assoc, v.221,n.12,p.1731-1736,2002. Disponívelem:<http://avmajournals. avma.org/doi/pdfplus/10.2460/javma.2002.221.1731>. Acesso em: 14 dez. 2012. doi: 10.2460/javma.2002.221.1731.

MISDORP, W. Canine mammary gland tumors: Protective effect of late ovariectomy and stimulating effect of progestins. Vet $\mathbf{Q}$, v.10, p.26-33, 1988. Disponível em: <http://www.tandfonline. com/doi/pdf/10.1080/01652176.1988.9694142>. Acesso em: 14 dez. 2012. doi: 10.1080/01652176.1988.9694142.

MORRISSON, W.B. Canine and feline mammary tumors. In MORRISSON, W.B. Cancer in dogs and cats. Baltimore: Williams \& Wilkins, 1998. p.591-598.

NGUYEN, P.G. et al. Effects of dietary fat and energy on body weight and composition after gonadectomy in cats. Am J Vet Res, v.65, p.1708-1713, 2004. Disponível em: <https://www.avma.org/ News/Journals/Collections/Documents/ajvr_65_12 1708.pdf>. Acesso em: 14 dez. 2012. doi: 10.2460/ajvr.2004.65.1708.

NICKEL, R.F. Studies on the function of the urethra and bladder in continent and incontinent female dogs. Vet Q, v.20, suppl.1, p.S102-103, 1998.

NIELSEN, J.C. et al. Effects of castration on problem behaviors in male dogs with reference to age and duration of behavior. $\mathbf{J ~ A m ~}$ Vet Med Assoc, v.211, p.180-182, 1997.

NORRIS, A.M. et al. Canine bladder and urethral tumors: a retrospective study of 115 cases (1980-1985). J Vet Intern Med, v.6, p.145-153, 1992. Disponível em: <http://onlinelibrary.wiley. com/doi/10.1111/j.1939-1676.1992.tb00330.x/pdf >. Acesso em: 18 dez. 2012. doi: 10.1111/j.1939-1676.1992.tb00330.x.

O'FARRELL, V.; PEACHEY, E. Behavioural effects of ovariohysterectomy on bitches. J Small Anim Pract, v.31, p.595-598, 1990. Disponível em: <http://onlinelibrary.wiley.com/ doi/10.1111/j.1748-5827.1990.tb00701.x/pdf>. Acesso em: 14 dez. 2012. doi: 10.1111/j.1748-5827.1990.tb00701.x.

OVERLEY, B. et al. Association between ovariohysterectomy and feline mammary carcinoma. J Vet Intern Med, v.19, p.560563, 2005. Disponível em: <http://onlinelibrary.wiley.com/ doi/10.1111/j.1939-1676.2005.tb02727.x/pdf>. Acesso em: 14 dez. 2012. doi: 10.1111/j.1939-1676.2005.tb02727.x.

PANCIERA, D.L. Hypothyroidism in dogs: 66 cases (1987-1992). J Am Vet Med Assoc, v.204, p.761-767, 1994.

PÖPPL, A.G. et al. Estudo preliminar da ligação hormônio-receptor da insulina à membranas de músculo e da tolerância à glicose em fêmeas caninas durante o ciclo estral. Acta Sci Vet, v.35, p.462464, 2007. Disponível em: <http://www.lume.ufrgs.br/bitstream/ handle $/ 10183 / 29226 / 000715799$.pdf? sequence $=1 \&$ locale $=$ en $>$. Acesso em: 14 dez. 2012.

PRAHL, A. et al. Time trends and risk factors for diabetes mellitus in cats presented to veterinary teaching hospitals. J Feline Med 
Surg, v.9, p.351-358, 2007. Disponível em: <http://jfm.sagepub. com/content/9/5/351.full.pdf $>$. Acesso em: 14 dez. 2012. doi: 10.1016/j.jfms.2007.02.004.

PRYMAK, C. et al. Epidemiologic, clinical, pathologic, and prognostic characteristics of splenic hemangiosarcoma and splenic hematoma in dogs: 217 cases (1985). J Am Vet Med Assoc, v.193, p.706-712, 1988 .

REICHLER, I.M. et al. FSH and LH plasma levels in bitches with differences in risk for urinary incontinence. Theriog, v.63, p.21642180, 2005. Disponível em: <http://pdn.sciencedirect.com/ science?_ob=MiamiImageURL\&_cid $=271179 \&$ user $=972052 \&$ pii $=$ S0093691X04003607\&_check=y\&_origin $=$ article\&_ zone $=$ toolbar\&_coverDate $=2005--31 \& v i e w=c \&$ originContent Family $=$ serial $\& w c h p=d G L z V 1 V-z S k W z \& m d 5=308$ ca370048 936b2489579279f6c605d\&pid=1-s2.0-S0093691X04003607main.pdf $>$. Acesso em: 14 dez. 2012. doi: 10.1016/j. theriogenology.2004.09.047.

REICHLER, I.M. et al. Urodynamic parameters and plasma LH/FSH in spayed Beagle bitches before and 8 weeks after GnRH depot analogue treatment. Theriog, v.66, p.21272136, 2006. Disponível em: <http://pdn.sciencedirect.com/ science? ob=MiamilmageURL\& cid $=271179 \&$ user $=972052 \&$ pii $=$ S0093691X0600358X\&_check $=y \&$ \&_origin $=$ article \&_ zone $=$ toolbar \& coverDate $=2006--31 \& v_{\text {view }}=$ c\&originConten tFamily $=$ serial $\&$ wchp $=$ dGLzV1S-zSkWz\&md5=971d0f2b 826 b50675ae262242fddb9a1\&pid=1-s2.0-S0093691X0600358Xmain.pdf>. Acesso em: 14 dez. 2012. doi: 10.1016/j. theriogenology.2006.06.009.

REICHLER, I.M. Gonadectomy in cats and dogs: a review of risks and benefits. Reprod Dom Anim, v.44, n.2, p.2935, 2009. Disponível em: <http://onlinelibrary.wiley.com/ store/10.1111/j.1439-0531.2009.01437.x/asset/j.1439. 0531.2009.01437.x.pdf? $\mathrm{v}=1 \& \mathrm{t}=$ haq2ao63\&s $=\mathrm{c} 9 \mathrm{eb} 002 \mathrm{e} 5626403$ $23 \mathrm{a} 0 \mathrm{e} 9939 \mathrm{~b} 4 \mathrm{~b} 01 \mathrm{ff} 5 \mathrm{~b} 149 \mathrm{ca} 13 \&$ system Message $=$ Wiley + Online + Library+will+be+disrupted +on+15+December+from $+10 \% 3 \mathrm{~A} 00$ $13 \% 3 \mathrm{~A} 00+\mathrm{GMT}+\% 2805 \% 3 \mathrm{~A} 00-08 \% 3 \mathrm{~A} 00+\mathrm{EST} \% 29+$ for + essent ial+maintenance>. Acesso em: 14 dez. 2012. doi: 10.1111/j.14390531.2009.01437.x.

REIMERS, T.J. et al. Effects of age, sex, and body size on serum concentrations of thyroid and adrenocortical hormones in dogs. Am J Vet Res, v.51, p.454-457, 1990.

RHODES L. et al. Estradiol causes a dose-dependent stimulation of prostate growth in castrated Beagle dogs. Prostate, v.44, p.8-18, 2000. Disponível em: <http://onlinelibrary.wiley. com/store/10.1002/1097-0045(20000615)44:1\%3C8::AIDPROS2\%3E3.0.CO;2-V/asset/2 ftp.pdf? $\mathrm{v}=1 \& \mathrm{t}=\mathrm{haq} 2 \mathrm{j} 6 \mathrm{ku} \& \mathrm{~s}=\mathrm{f} 5 \mathrm{~d}$ 4 fa 41 ce 2 b890b63fabd56afff7a 1 e 32 b49c3a\&systemMessage $=$ Wil ey+Online + Library + will + be + disrupted + on $+15+$ December + from $+10 \% 3 \mathrm{~A} 00-13 \% 3 \mathrm{~A} 00+\mathrm{GMT}+\% 2805 \% 3 \mathrm{~A} 00-08 \% 3 \mathrm{~A} 00+\mathrm{EST} \% 2$ 9+for+essential+maintenance $>$. Acesso em: $14 \mathrm{dez}$. 2012. doi: 10.1002/1097-0045(20000615)44:1<8::AID-PROS2>3.0.CO;2-V.

RIGGS, B.L. et al. Sex steroids and the construction and conservation of the adult skeleton. Endocr Rev, v.23, p.279302, 2002. Disponível em: <http://edrv.endojournals.org/ content/23/3/279.full.pdf $>$. Acesso em: 14 dez. 2012 . doi: 10.1210/er.23.3.279.

ROOT, M.V. et al. Effect of prepubertal and postpubertal gonadectomy on heat production measured by indirect calorimetry in male and female domestic cats. Am J Vet Res, v.57, p.371-374, 1996a.
ROOT, M.V. et al. The effects of prepubertal and postpubertal gonadectomy on penile extrusion and urethral diameter in the domestic cat. Vet Radiol Ultrasound, v.37, p.363-366, 1996b. Disponível em: <http://onlinelibrary.wiley.com/ doi/10.1111/j.1740-8261.1996.tb01244.x/pdf>. Acesso em: 18 dez. 2012. doi: 10.1111/j.1740-8261.1996.tb01244.x.

ROOT, M.V. et al. The effect of prepuberal and postpuberal gonadectomy on radial physeal closure in male and female domestic cats. Vet Radiol Ultrasound, v.38, p.42-47, 1997. Disponível em: $<$ http://onlinelibrary.wiley.com/store/10.1111/j.1740-8261.1997. tb01601.x/asset/j.1740-8261.1997.tb01601.x.pdf? $\mathrm{v}=1 \& \mathrm{t}=\mathrm{haq} 3 \mathrm{k}$ 0mn\&s $=1 \mathrm{a} 182871 \mathrm{bcb} 2 \mathrm{~b} 5986 \mathrm{afa} 77 \mathrm{da} 8 \mathrm{c} 8 \mathrm{bf} 269 \mathrm{bfe} 27564 \&$ syste mMessage $=$ Wiley + Online + Library + will + be + disrupted + on $+15+$ December+from $+10 \% 3 \mathrm{~A} 00-13 \% 3 \mathrm{~A} 00+\mathrm{GMT}+\% 2805 \% 3 \mathrm{~A} 00-$ 08\%3A00+EST\%29+for+essential+maintenance>. Acesso em: 14 dez. 2012. doi: 10.1111/j.1740-8261.1997.tb01601.x.

RU, G. et al. Host related risk factors for canine osteosarcoma. Vet J, v.156, p.31-39, 1998. Disponível em: <http://ac.els-cdn. com/S1090023398800592/1-s2.0-S1090023398800592-main. pdf? tid=530b4626-465b-11e2-8abe-00000aab0f $27 \&$ acdnat $=135$ 5537062_72b66c3b1ce5b498c0392f8307f1b955>. Acesso em: 14 dez. 2012. doi: 10.1016/S1090-0233(98)80059-2.

RUTTEMAN, G.R. et al. Tumors of the mammary gland. In: WITHROW, S.J.; MACEWEN, E.G. Small animal clinical oncology. Philadelphia: Saunders, 2001. p.455-477.

SALMERI, K.R. et al. Gonadectomy in immature dogs: effects on skeletal, physical, and behavioral development. J Am Vet Med Assoc, v.198, p.1193-1203, 1991.

SCARAMAL, J.D. et al. Natural estrous cycle in normal and diabetic bitches in relation to glucose and insulin tests. Medicina (Buenos Aires), v.57, p.169-180, 1997.

SCOTT-MONCRIEFF, J.C. Insulin resistance in cats. Vet Clin Small Anim, v.40, p.241-257, 2010. Disponível em: <http:// www.2ndchance.info/diabetescat-insulinresistance.pdf $>$. Acesso em: 18 dez. 2012. doi: 10.1016/j.cvsm.2009.10.007.

SCHNEIDER, R. et al. Factors influencing canine mammary cancer development and postsurgical survival. J Natl Cancer Inst, v.43, p.1249-1261, 1969. Disponível em: <http://jnci. oxfordjournals.org/content/43/6/1249.full.pdf>. Acesso em: 14 dez. 2012. doi: 10.1093/jnci/43.6.1249.

SCHOEMAKER, N.J. et al. Correlation between age at neutering and age at onset of hyperadrenocorticism in ferrets. J Am Vet Med Assoc, v.216, p.195-197, 2000. Disponível em: <http:// avmajournals.avma.org/doi/pdf/10.2460/javma.2000.216.195>. Acesso em: 18 dez. 2012. doi: 10.2460/javma.2000.216.195.

SHAHIDI, N.T. A review of the chemistry, biological action, and clinical applications of anabolic-androgenic steroids. Clin Ther, v.23, n.9, p.1355-1390, 2001. Disponível em: <http://ac.els-cdn. com/S0149291801801144/1-s2.0-S0149291801801144-main. pdf? tid=a9233846-465d-11e2-99fb-00000aab0f01\&acdnat $=135$ 5538065_593d0dfbf5d91f6052684a6dea58a600>. Acesso em: 14 dez. 2012. doi: 10.1016/S0149-2918(01)80114-4.

SHEN, V. et al. Lack of changes in histomorphometric, bone mass, and biochemical parameters in ovariohysterectomized dogs. Bone, v.13, p.311-316, 1992. Disponível em: < http://ac.els-cdn. com/8756328292900758/1-s2.0-8756328292900758-main.pdf? 
tid=10058672-465e-11e2-a9f0-00000aab0f6c\&acdnat $=13555382$ 38 63d2d4a2e832c21616569b5dec1cd8c0>. Acesso em: $14 \mathrm{dez}$. 2012. doi: 10.1016/8756-3282(92)90075-8.

SLAUTERBECK, J.R. et al. Canine ovariohysterectomy and orchiectomy increases the prevalence of ACL injury. Clin Orthop Relat Res, v.429, p.301-305, 2004.

SONNENSCHEIN, E.G. et al. Body conformation, diet, and risk of breast cancer in pet dogs: a case-control study. Am J Epidemiol, v.133, p.694-703, 1991. Disponível em: <http://aje.oxfordjournals. org/content/133/7/694.full.pdf>. Acesso em: 14 dez. 2012.

SONTAS, B.H.; EKICI, H. Short-term effects of prepubertal ovariohysterectomy on skeletal, physical and behavioural development of dogs up to 24 weeks of age. Acta Vet Hung, v.55, n.3, p.379-387, 2007. Disponível em: <http://www.akademiai.com/content/752v772j0751u042/ fulltext.pdf $>$. Acesso em: 14 dez. 2012. doi: 10.1556/ AVet.55.2007.3.12.

SORENMO, K.U. et al. Effect of spaying and timing of spaying on survival of dogs with mammary carcinoma. J Vet Intern Med, v.14, p.266-270, 2000. Disponível em: $<$ http://onlinelibrary. wiley.com/store/10.1111/j.1939-1676.2000.tb01165.x/ asset/j.1939-1676.2000.tb01165.x.pdf? $\mathrm{v}=1 \& \mathrm{t}=$ havmgtip $\& \mathrm{~s}=571 \mathrm{e}$ dcd5c7f55d501b6867222edecea4b92b54c3>. Acesso em: $18 \mathrm{dez}$. 2012. doi: 10.1111/j.1939-1676.2000.tb01165.x.

SORENMO, K.U. et al. Immunohistochemical characterization of canine prostatic carcinoma and correlation with castration status and time. Vet Comp Oncol, v.1, p.4856, 2003. Disponível em: <http://onlinelibrary.wiley.com/ store/10.1046/j.1476-5829.2003.00007.x/asset/j.14765829.2003.00007.x.pdf? $\mathrm{v}=1 \& \mathrm{t}=$ haq5 $\mathrm{cwld} \& \mathrm{~s}=6 \mathrm{c} 59 \mathrm{~d} 6 \mathrm{~d} 9 \mathrm{e} 1 \mathrm{db} 6 \mathrm{cc}$ 6a0b1986b1566ac925bfc475c\&systemMessage $=$ Wiley + Online + Library+will+be+disrupted +on $+15+$ December + from $+10 \% 3 \mathrm{~A} 00$ $13 \% 3 \mathrm{~A} 00+\mathrm{GMT}+\% 2805 \% 3 \mathrm{~A} 00-08 \% 3 \mathrm{~A} 00+\mathrm{EST} \% 29+$ for + essen tial+maintenance $>$. Acesso em: 14 dez. 2012. doi: 10.1046/j.14765829.2003.00007.x.

SPAIN, C.V. et al. Long-term risks and benefits of early-age gonadectomy in dogs. J Am Vet Med Assoc, v.224, p.380-387, 2004a. Disponível em: <http://avmajournals.avma.org/doi/ pdf/10.2460/javma.2004.224.380>. Acesso em: 14 dez. 2012. doi: 10.2460/javma.2004.224.380.

SPAIN, C.V. et al. Long-term risks and benefits of early-age gonadectomy in cats. J Am Vet Med Assoc, v.224, p.372-379, 2004b. Disponível em: <http://avmajournals.avma.org/doi/ pdf/10.2460/javma.2004.224.372>. Acesso em: 14 dez. 2012. doi: 10.2460/javma.2004.224.372.

STÖCKLIN-GAUTSCHI, N.M. et al. The relationship of urinary incontinence to early spaying in bitches. J Rep Fertil Suppl, v.57, p.233-236, 2001 .

STUBBS, W.P. et al. Effects of prepubertal gonadectomy on physical and behavioral development in cats. J Am Vet Med Assoc, v.209, p.1864-1871, 1996.

SYED, F.; KHOSLA, S. Mechanisms of sex steroid effects on bone. Biochem Biophys Res Commun, v.328, p.688-696, 2005. Disponível em: <http:/ac.els-cdn.com/S0006291X04026415/1s2.0-S0006291X04026415-main.pdf? tid=92e $0044 \mathrm{a}-$ 4664-11e2-86c2-00000aacb362\&acdnat $=1355541035$ b05f05a5d74fab3e7c7826e8f7584297>. Acesso em: 14 dez. 2012. doi: 10.1016/j.bbrc.2004.11.097.

TESKE, E. et al. Canine prostate carcinoma: epidemiological evidence of an increased risk in castrated dogs. Mol Cell Endocrinol, v.197, p.251-255, 2002. Disponível em: $<$ http://ac.elscdn.com/S0303720702002617/1-s2.0-S0303720702002617-main. pdf?_tid=96a03202-4665-11e2-b969-00000aab0f27\&acdnat=13 55541470_4970cba779a55259d443464aa2cb4451>. Acesso em: 14 dez. 2012. doi: 10.1016/S0303-7207(02)00261-7.

THRUSFIELD, M.V. et al. Acquired urinary incontinence in bitches: its incidence and relationship to neutering practices. $\mathbf{J}$ Small Anim Pract, v.39, p.559-566, 1998. Disponível em: $<$ http://onlinelibrary.wiley.com/store/10.1111/j.1748-5827.1998. tb03709.x/asset/j.1748-5827.1998.tb03709.x.pdf? $v=1 \& \mathrm{t}=$ haq6d 87m\&s=b9caa18da4e30d277290123b827c93a332412a82\&syst emMessage $=$ Wiley + Online + Library + will + be + disrupted + on +15 + December+from + 10\%3A00-13\%3A00+GMT+\%2805\%3A0008\%3A00+EST\%29+for+essential+maintenance>. Acesso em: 14 dez. 2012. doi: 10.1111/j.1748-5827.1998.tb03709.x.

VAN HAGEN, M.A. et al. Incidence, risk factors, and heritability estimates of hind limb lameness caused by hip dysplasia in a birth cohort of boxers. Am J Vet Res, v.66, p.307-312, 2005. Disponível em: <http://avmajournals.avma.org/doi/pdf/10.2460/ ajvr.2005.66.307>. Acesso em: 14 dez. 2012. doi: 10.2460/ ajvr.2005.66.307.

WARE, W.A.; HOPPER, D.L. Cardiac tumors in dogs: 1982 1995. J Vet Intern Med, v.13, p.95-103, 1999. Disponível em: $<$ http://radfileshare.cvm.ncsu.edu/RIS/STUFF/Case\%20of\%20 vertebral\%20tumor/Cardiac\%20tumors\%20in\%20dogs.pdf>. Acesso em: 18 dez. 2012. doi: 10.1111/j.1939-1676.1999.tb01136.x.

Ciência Rural, v.43, n.6, jun, 2013. 